\title{
Axiom System and Some Theorems for Dialectical-Logic K-Model
}

\author{
Yaozhi Jiang \\ Shijiazhuang HighTec Zone, Hebei, China \\ Email: jiangyaozhi@126.com
}

How to cite this paper: Jiang, Y.Z. (2017) Axiom System and Some Theorems for Dialectical-Logic K-Model. Open Access Library Journal, 4: e3817.

https://doi.org/10.4236/oalib.1103817

Received: July 11, 2017

Accepted: August 25, 2017

Published: August 28, 2017

Copyright (c) 2017 by author and Open Access Library Inc.

This work is licensed under the Creative Commons Attribution International License (CC BY 4.0).

http://creativecommons.org/licenses/by/4.0/

\begin{abstract}
An axiom system for dialectical logic K-model which is based on energy-method is established by author in the paper. Dialectical logic K-model supplies a computation-idea for machine, so that it can be applied in many computations for artificial intelligence. This paper described that subjective-laws is the mirror imagine reflected from objective-laws, and defined the three-step which is named by sensation, abstraction and thinking separately in artificial intelligence. At the same time, the author described axiom system for dialectical logic K-model which contains logic-variable energy conservation law, Mozi-principle (minimax principle) and forbidden law, etc. In the axiom system, it also contains a continuous-true-value function valued on interval [-1, +1 , and the K-graph for logic-variable. Dialectical logic K-model would supply a computation-idea for machine so that the machine is able to think by dialectical logic method, thus an important information-treated method may be the dialectical logic.
\end{abstract}

\section{Subject Areas}

Mathematical Logic and Foundation of Mathematics

\section{Keywords}

Artificial Intelligence, Dialectical Logic K-Model, K-Graph $G_{K}$, Kirchoff-Laws, Contradiction-Function

\section{Introduction}

Objective world consists of five factors which are MATTER, ENERGY, INFORMATION, SPACE and TIME. Among these factors, there are many inner or outer laws of causation called objective laws, which can also be called as objective logic. Corresponding to objective laws, there is a mirror image in machine of artificial intelligence, which can be called subjective laws, and also can 
be called as subjective logic. The machine of artificial intelligence only holds objective laws through subjective laws, or with another word, only holds objective logic through subjective logic. Subjective logic is built on infinite times circulation by the three-step named Sensation, Abstraction and Thinking. Sensation is input-ability to machine from objective information with sensor. Abstraction is conception-classification ability to sensed information and to name it. Thinking is the ability of machine researching from conception-classification information to get the subjective logic.

Dialectical logic K-model would supply a computation-idea for machine so that the machine is able to think by dialectical logic method [1] [2], so an important information-treated method would be the dialectical logic. In this paper now, author has built a mathematical model for dialectical logic, and the model combines with three main identification technologies (identification technology of graph, identification technology of sound, identification technology of written language), and data-base technology and machine-self-programming technology will take a great progress to artificial intelligence.

\section{Axiom System and Theorems}

\subsection{Conception and the Conception-Dimension}

\subsubsection{The Conception}

The conception is the name what be named the object-thing's main property and neglected its secondary property. Conception is the basis of artificial intelligence.

\subsubsection{The conception-Dimension}

The conception-dimension is the dimension-number of conception. For example, HORSE is an one-dimension conception; WHITE HORSE is a two-dimension conception; RUNNING WHITE HORSE is a three-dimension conception; ONE RUNNING WHITE HORSE is a four-dimension conception; etc.

\subsubsection{Associate-Data-Base}

The three main identification technologies will setup three data-base, although there are other data-base, for example physical data-base and chemical database, etc. These three data-bases are associated by the conception-dimension.

\subsection{Several Laws}

\subsubsection{Definitions}

Denote objective-demain by $B$, and subjective-demain by $A$; define researcharithmetic $\nabla$ (research-arithmetic is an arithmetic to get subjective-logic from objective-logic) and inverse research-arithmetic $\nabla^{-1}$ (inverse research-arithmetic is an arithmetic to make subjective-logic to objective-logic to check its true or false) and thus

$$
\begin{aligned}
& \nabla\left(B_{i} \in B\right) \Leftrightarrow \nabla\left(A_{i} \in A\right) \\
& \nabla^{-1} \nabla\left(B_{i} \in B\right) \Leftrightarrow \nabla^{-1} \nabla\left(A_{i} \in A\right) \\
& \cdots \\
& \nabla^{-1} \nabla \cdots \nabla^{-1} \nabla\left(B_{i} \in B\right) \Leftrightarrow \nabla^{-1} \nabla \cdots \nabla^{-1} \nabla\left(A_{i} \in A\right)
\end{aligned}
$$




$$
\begin{aligned}
& \nabla^{-1} \nabla=F_{1} \\
& \cdots \\
& \underbrace{\nabla^{-1} \nabla \cdots \nabla^{-1} \nabla \nabla^{-1} \nabla}_{j \text { couples of } \nabla^{-1} \nabla}=F_{j}
\end{aligned}
$$

Then $F_{j}\left(B_{i} \in B\right) \Leftrightarrow F_{j}\left(A_{i} \in A\right)$

As above, $F_{j}$ is the $j$-th cycle research-arithmetic, obviously $F_{j}$ is a logic-transformation between demain $A$ and demain $B$. Sometimes can denote $F_{j}$ by $F$. Symbol $\Leftrightarrow$ is denote the object logic and the subject logic are mirror imagine each other.

Denote subjective-logic-variable by

$$
A_{i}^{ \pm n}(t)=\left\{a_{1}^{ \pm}(t), a_{2}^{ \pm}(t), \cdots, a_{n}^{ \pm}(t)\right\}
$$

as above, $t$ is time-variable, right superscript $\pm n$ denote the logic-variable contains $n$ couples of contradicting-subvariable $a_{i}^{ \pm}(t), n$ is called the rank-number of $A_{i}^{ \pm n}(t)$, right subscript $i$ denote the $i$-th of causation law.

$T\left(A_{i}^{ \pm n}(t)\right)$ is denoted the true-valued-function of logic-variable $A_{i}^{ \pm n}(t)$, and $T\left(A_{i}^{ \pm n}(t)\right) \in[-1,1]$.

Denote the mirror-image in objective-demain of $A_{i}^{ \pm n}(t) \in A$ by $B_{i}(t) \in B$.

\subsubsection{Objective-Researchable Law}

To every object logic-variable $B_{i}(t) \in B$, there must always exist logic-transformation $F$, make

$$
A_{i}^{ \pm n}(t)=F\left(B_{i}(t)\right)
$$

\subsubsection{Research Error Alternating-Convergence Law}

To every correct logic-transformation $F$, always make

$$
\lim _{j \rightarrow \infty} C\left|A_{i}^{ \pm n}(t)-B_{i}(t)\right|=\lim _{j \rightarrow \infty} C\left|F_{j}\left(B_{i}(t)\right)-B_{i}(t)\right|=0
$$

i.e. to every finite $j<\infty$, error-function

$$
E_{j}(t)=\left|F_{j}\left(B_{i}(t)\right)-B_{i}(t)\right|>0
$$

In the formulation as above $C=+1$, if $j=2 p+1$; or $C=-1$, if $j=2 p$, $p=0,1,2, \cdots$

In fact, incorrect logic-transformation $F^{*}$ can bring the researching divergence. The faster is the convergence of $F_{j}$, the brighter is the $F_{j}$.

\subsubsection{Logic-Variable Energy Conservation Law}

For logic-variable $A_{i}^{ \pm n}(t)$, its inner producing-energy $E_{i}$ is equal to the consumed work $J_{i}$ by its contradiction-function (see the 10.4 contradiction-function as below), i.e.

$$
E_{i}=J_{i}
$$

\subsubsection{Mozi-Principle (Minimax Principle) [3]}

Logic-variable $A_{i}^{ \pm n}(t)$ in changing, must be satisfied or must be satisfied asymp- 
totically by that cost-function $C\left(A_{i}^{ \pm n}(t)\right)$ is minimum and gain-function $G\left(A_{i}^{ \pm n}(t)\right)$ is maximum. Denote the pure gain-function by $L\left(A_{i}^{ \pm n}(t)\right)$, i.e.

$$
L\left(A_{i}^{ \pm n}(t)\right)=\max \left(G\left(A_{i}^{ \pm n}(t)\right)-\min C\left(A_{i}^{ \pm n}(t)\right)\right)
$$

or

$$
\lim _{t \rightarrow \infty} L\left(A_{i}^{ \pm n}(t)\right)=\max _{t \rightarrow \infty}\left(G\left(A_{i}^{ \pm n}(t)\right)-\min _{t \rightarrow \infty} C\left(A_{i}^{ \pm n}(t)\right)\right)
$$

\subsubsection{Memory-Inertia Law}

1) Last-time memory law

For time-sequence $t_{1}<t_{2}<\cdots<t_{k}<\cdots$, denote the memory effect-weight-function of contradiction-subvariable $a_{i}^{ \pm}\left(t_{p}\right)$ from time t by $R\left(a_{i}^{ \pm}\left(t_{p}\right)\right)$,

$$
R\left(a_{i}^{ \pm}\left(t_{p}\right)\right) \in[0,1]
$$

and $R\left(a_{i}^{ \pm}\left(t_{k}\right)\right)>R\left(a_{i}^{ \pm}\left(t_{j}\right)\right)$

If and only if $t_{k}>t_{j}$. Memory prefer the $a_{k}^{ \pm}(t)$ of the last time.

2) Importance memory law

For importance-sequence $M\left(a_{p}^{ \pm}(t)\right)<M\left(a_{q}^{ \pm}(t)\right)<\cdots<M\left(a_{r}^{ \pm}(t)\right)$, and $M\left(a_{i}^{ \pm}(t)\right) \in[0,1]$

So the memory prefer the $a_{r}^{ \pm}(t)$, if and only if its $M\left(a_{r}^{ \pm}(t)\right)$ is bigger.

3) Bigger probability memory law

For property event $\alpha_{1}, \alpha_{2}, \cdots, \alpha_{k}$, their corresponding probability are

$$
P\left(\alpha_{1}\right), P\left(\alpha_{2}\right), \cdots, P\left(\alpha_{k}\right) \text {, }
$$

and

$$
P\left(\alpha_{P}\right)>P\left(\alpha_{q}\right)
$$

so the memory prefer $\alpha_{p}$.

\subsection{Machine Self-Programmable and Self-Correctable Law}

In the researching process the machine must have ability to self-programmable and self-correctable without the operations by human beings.

\subsection{Forbidden Law}

There are two kinds of logic: one is dialectical-logic corresponding to intelligence quotient and another is imagine-logic corresponding to emotional quotient.

The imagine-logic would be forbidden into machine, because the imagine-logic will make machine to emotional quotient so that the autonomous-mind will belong to the machine. The artificial intelligence with the autonomous-mind will not like to be "a tool" for human beings, so "a new creation" will be created, of cause this is harmful to human beings.

\subsection{Logic-Variable Infinite-Separable-Characteristic Law}

$$
A_{i}^{ \pm n}(t)=\lim _{n \rightarrow \infty}\left\{a_{1}^{ \pm}(t), a_{2}^{ \pm}(t), \cdots, a_{n}^{ \pm}(t)\right\}
$$




\subsection{Logical Inductive and Deductive Method Theorem}

For cycle research-arithmetic $F_{j}$, make

$$
A_{i}^{ \pm n}(t)=F_{j}\left(B_{i}(t)\right)
$$

1) (inductive) if as above is true to finite $F_{k}, k=1,2, \cdots, j$

2) (hypothesis) suppose as above is true to $F_{k+1}$,

3) (deductive) so that as above is true to all $j<\infty$.

Proof: through finite to prove infinite, there always exists error-function based on the 2.3. research error alternating-convergence law, if the 6.2. (hypothesis) is false, then producing the false to the 6.3. (deductive). Thus the truth to every step of $F_{j}$ would be carefully checked.

Proof is over.

\subsection{Definitions of Logic Algorithm}

For $A_{i}^{ \pm r}(t), A_{i}^{ \pm s}(t), A_{i}^{ \pm k}(t)$, denote their true-valued-function by corresponding to $T\left(A_{i}^{ \pm r}(t)\right), T\left(A_{i}^{ \pm s}(t)\right), T\left(A_{i}^{ \pm k}(t)\right)$,

\subsubsection{Logic $U$ (OR Arithmetic)}

Definition $T\left(A_{i}^{ \pm s}(t)\right) \cup T\left(A_{i}^{ \pm r}(t)\right)=\max \left\{T\left(A_{i}^{ \pm s}(t)\right) ; T\left(A_{i}^{ \pm r}(t)\right)\right\} \quad$ logic $\bigcup$ is satisfied by as below

Commutative law $T\left(A_{i}^{ \pm s}(t)\right) \cup T\left(A_{i}^{ \pm r}(t)\right)=T\left(A_{i}^{ \pm r}(t)\right) \cup T\left(A_{i}^{ \pm s}(t)\right)$ associative law

$$
\left(T\left(A_{i}^{ \pm s}(t)\right) \cup T\left(A_{i}^{ \pm r}(t)\right)\right) \cup T\left(A_{i}^{ \pm k}(t)\right)=T\left(A_{i}^{ \pm s}(t)\right) \cup\left(T\left(A_{i}^{ \pm r}(t)\right) \cup T\left(A_{i}^{ \pm k}(t)\right)\right)
$$

\subsubsection{Logic $\cap$ (AND Arithmetic)}

Definition $T\left(A_{i}^{ \pm s}(t)\right) \cap T\left(A_{i}^{ \pm r}(t)\right)=\min \left\{T\left(A_{i}^{ \pm s}(t)\right) ; T\left(A_{i}^{ \pm r}(t)\right)\right\} \quad$ logic $\bigcap$ is satisfied by as below commutative law

$$
\begin{aligned}
& T\left(A_{i}^{ \pm s}(t)\right) \cap T\left(A_{i}^{ \pm r}(t)\right)=T\left(A_{i}^{ \pm r}(t)\right) \cap T\left(A_{i}^{ \pm s}(t)\right) \text { associative law } \\
& \left(T\left(A_{i}^{ \pm s}(t)\right) \cap T\left(A_{i}^{ \pm r}(t)\right)\right) \cap T\left(A_{i}^{ \pm k}(t)\right)=T\left(A_{i}^{ \pm s}(t)\right) \cap\left(T\left(A_{i}^{ \pm r}(t)\right) \cap T\left(A_{i}^{ \pm k}(t)\right)\right)
\end{aligned}
$$

\subsubsection{Logic Hybrid Arithmetic (OR \& AND)}

Logic hybrid arithmetic is satisfied as below

Distributive law

$$
\begin{aligned}
& T\left(A_{i}^{ \pm s}(t)\right) \cup\left(T\left(A_{i}^{ \pm r}(t)\right) \cap T\left(A_{i}^{ \pm k}(t)\right)\right) \\
& =\left(T\left(A_{i}^{ \pm s}(t)\right) \cup T\left(A_{i}^{ \pm r}(t)\right)\right) \cap\left(T\left(A_{i}^{ \pm s}(t)\right) \cup T\left(A_{i}^{ \pm k}(t)\right)\right)
\end{aligned}
$$

\subsubsection{Logic $N$ (NOT Arithmetic)}

$N\left[T\left(A_{i}^{ \pm n}(t)\right)\right]= \pm 1-T\left(A_{i}^{ \pm n}(t)\right)$, if $T\left(A_{i}^{ \pm n}(t)\right)>0$ is positive in front of the 1 ; if $T\left(A_{i}^{ \pm n}(t)\right)<0$ is negative in front of the 1.

Denote $\underbrace{N\left[N\left[\cdots N\left[A_{i}^{ \pm n}(t)\right]\right]\right]}_{p}=N^{p}\left[A_{i}^{ \pm n}(t)\right]$ 
Arithmetic $N$ is satisfied by as below idempotent law $N^{p}\left[A_{i}^{ \pm n}(t)\right]=A_{i}^{ \pm n}(t)$, if $p=2 k ; N^{p}\left[A_{i}^{ \pm n}(t)\right]=N\left[A_{i}^{ \pm n}(t)\right]$, if $p=2 k+1 ; k=0,1,2, \cdots$

\subsection{De Morgan's Theorem}

$$
\begin{aligned}
& N\left[T\left(A_{i}^{ \pm s}(t) \cap T\left(A_{i}^{ \pm r}(t)\right)\right)\right]=N\left[T\left(A_{i}^{ \pm s}(t)\right)\right] \cup N\left[T\left(A_{i}^{ \pm r}(t)\right)\right] \\
& N\left[T\left(A_{i}^{ \pm s}(t)\right) \cup T\left(A_{i}^{ \pm r}(t)\right)\right]=N\left[T\left(A_{i}^{ \pm s}(t)\right)\right] \cap N\left[T\left(A_{i}^{ \pm r}(t)\right)\right]
\end{aligned}
$$

Proof:

$$
\begin{aligned}
N\left[T\left(A_{i}^{ \pm s}(t)\right) \cap T\left(A_{i}^{ \pm r}(t)\right)\right] & = \pm 1-\left(T\left(A_{i}^{ \pm s}(t)\right) \cap T\left(A_{i}^{ \pm r}(t)\right)\right) \\
& = \pm 1-\min \left\{T\left(A_{i}^{ \pm s}(t)\right) ; T\left(A_{i}^{ \pm r}(t)\right)\right\} \\
& =\max \left\{ \pm 1-T\left(A_{i}^{ \pm s}(t)\right) ; \pm 1-T\left(A_{i}^{ \pm r}(t)\right)\right\} \\
& =N\left[T\left(A_{i}^{ \pm s}(t)\right)\right] \bigcup\left[T\left(A_{i}^{ \pm r}(t)\right)\right]
\end{aligned}
$$

Formulation (1) proof is over.

$$
\begin{aligned}
N\left[T\left(A_{i}^{ \pm s}(t)\right) \cup T\left(A_{i}^{ \pm r}(t)\right)\right] & = \pm 1-\left(T\left(A_{i}^{ \pm s}(t)\right) \cup T\left(A_{i}^{ \pm r}(t)\right)\right) \\
& = \pm 1-\max \left\{T\left(A_{i}^{ \pm s}(t)\right) ; T\left(A_{i}^{ \pm r}(t)\right)\right\} \\
& =\min \left\{ \pm 1-T\left(A_{i}^{ \pm s}(t)\right) ; \pm 1-T\left(A_{i}^{ \pm r}(t)\right)\right\} \\
& =\min \left\{N\left[T\left[A_{i}^{ \pm s}(t)\right]\right] ; N\left[T\left[A_{i}^{ \pm r}(t)\right]\right]\right\} \\
& =N\left[T\left(A_{i}^{ \pm s}(t)\right)\right] \cap N\left[T\left(A_{i}^{ \pm r}(t)\right)\right]
\end{aligned}
$$

Formulation (2) proof is over.

Remark 1: As above when time $t$ is degenerated into a constant and destroy the contradictions in the formulations, then the formulations will be degenerated into the mathematical model of formal logic, i.e. Boolean algebra.

\subsection{Logic Algorithm True-Valued-Function Composition Theorem}

For logic arithmetic of true-valued-function $T\left(A_{i}^{ \pm s}(t)\right), T\left(A_{i}^{ \pm r}(t)\right)$, as below there is a composition theorem:

If equation $T\left(A_{i}^{ \pm s}(t)\right)-T\left(A_{i}^{ \pm r}(t)\right)=0$ exist root-set

$T=\left\{t_{1}, t_{2}, \cdots, t_{m}\right\} \in\left[t_{0}, t\right]$, and $t_{1}<t_{2}<\cdots<t_{m}$ so that

$$
\begin{aligned}
& \max \left(T\left(A_{i}^{ \pm s}(t)\right) ; T\left(A_{i}^{ \pm r}(t)\right)\right) \\
& =\max _{t \in\left[t_{0}, t_{1}\right]}\left(T\left(A_{i}^{ \pm s}(t) ; T\left(A_{i}^{ \pm r}(t)\right)\right)\right)+\max _{t \in\left[t_{1}, t_{2}\right]}\left(T\left(A_{i}^{ \pm s}(t)\right) ; T\left(A_{i}^{ \pm r}(t)\right)\right) \\
& \quad+\max _{t \in\left[t_{2}, t_{3}\right]}\left(T\left(A_{i}^{ \pm s}(t)\right) ; T\left(A_{i}^{ \pm r}(t)\right)\right)+\cdots+\max _{t \in\left[t_{m}, t_{3}\right]}\left(T\left(A_{i}^{ \pm s}(t)\right) ; T\left(A_{i}^{ \pm r}(t)\right)\right)
\end{aligned}
$$

as same as above, also

$$
\begin{aligned}
& \min \left(T\left(A_{i}^{ \pm s}(t)\right) ; T\left(A_{i}^{ \pm r}(t)\right)\right) \\
& =\min _{t \in\left[t_{0}, t_{1}\right]}\left(T\left(A_{i}^{ \pm s}(t)\right) ; T\left(A_{i}^{ \pm r}(t)\right)\right)+\min _{t \in\left[t_{1}, t_{2}\right]}\left(T\left(A_{i}^{ \pm s}(t)\right) ; T\left(A_{i}^{ \pm r}(t)\right)\right) \\
& \quad+\min _{t \in\left[t_{2}, t_{3}\right]}\left(T\left(A_{i}^{ \pm s}(t)\right) ; T\left(A_{i}^{ \pm r}(t)\right)\right)+\cdots+\min _{t \in\left[t_{m}, t\right]}\left(T\left(A_{i}^{ \pm s}(t)\right) ; T\left(A_{i}^{ \pm r}(t)\right)\right)
\end{aligned}
$$


If equation $T\left(A_{i}^{ \pm s}(t)\right)-T\left(A_{i}^{ \pm r}(t)\right)=0$ in which the possible augment root and complex root will be removed out.

If $T\left(A_{i}^{ \pm s}(t)\right)-T\left(A_{i}^{ \pm r}(t)\right) \neq 0$, so the root-set $T=\varnothing$ that

$\max \left(T\left(A_{i}^{ \pm s}(t)\right) ; T\left(A_{i}^{ \pm r}(t)\right)\right)$ or $\min \left(T\left(A_{i}^{ \pm s}(t)\right) ; T\left(A_{i}^{ \pm r}(t)\right)\right)$ in which select a maximum or minimum of pure function.

Proof: obviously based on definition of logic arithmetic.

Proof is over.

\subsection{Kirchoff's Power-Function Law and Kirchoff's Flow-Function Law [4]}

Defines a connecting directed simple graph $G_{K}$ with $2(n+1)$ nodes and $\mathrm{E}$ edges $G_{K}=\left\{V_{2(n+1)} ; E\right\}$

In graph $G_{K}$ every node will be given a power-function $v_{i}(t)$; for node $V_{i}$ and node $V_{j}$, if edge $E_{i j} \in E$, then on the edge $E_{i j}$ exists a power-function

$$
e_{i j}(t)=v_{j}(t)-v_{i}(t)
$$

In Formulation (3), lower reaches node is left and upper reaches node is right, in opposite direction would sign a negative in the front of $e_{i j}(t)$.

In graph $G_{K}$ if the edge $E_{i j}$ exists, then give a flow-function $f_{i j}(t)$, its direction is from the upper reaches node to the lower reaches node. The graph $G_{K}$ would be satisfied by 10.2., 10.3., 10.4., 10.5. as below.

\subsubsection{Kirchoff's Power-Function Law}

In the graph $G_{K}$ every cycle $W_{i j}$ is satisfied by

$$
\sum_{\text {if } E_{i j} \in W_{i j}} e_{i j}(t)=0
$$

\subsubsection{Kirchoff's Flow-Function Law}

In the graph $G_{K}$ every node $V_{i}$ is satisfied by

$$
\sum_{\text {if } E_{i} \in V_{i}} f_{i}(t)=0
$$

as above input flow $f_{i}(t)$ is positive and output flow $f_{i}(t)$ is negative.

\subsubsection{Contradiction-Function}

Defines

$$
R_{i j}(t)=\frac{e_{i j}(t)}{f_{i j}(t)}
$$

is the contradiction-function of dge $E_{i j}$ in graph $G_{K}$.

\subsubsection{Work and Energy Law}

In the graph $G_{K}$ every edge $E_{i j}$, make

$$
W_{i j}=\int_{t_{0}}^{t_{1}} e_{i j}(t) f_{i j}(t) \mathrm{d} t
$$

$W_{i j}$ is called what work done in $\left[t_{0}, t_{1}\right]$ by contradiction-function $R_{i j}(t)$ on edge $E_{i j}$. 


\subsection{Structure in Graph $G_{K}$ of Logic-Variable $A_{i}^{ \pm n}(t)$}

\section{(Figure 1)}

1) Graph $G_{K}$ have $\mathrm{n}$ couples of nodes and two nodes $V_{+1}, V_{-1}$, total $2(n+1)$;

2) Graph $G_{K}$ is a no-loop, no-multiple edge directed simple graph;

3) In the graph $G_{K}, n+1$ positive nodes construct a perfect subgraph, another $\mathrm{n}+1$ negative node construct another perfect subgraph;

4) Node $V_{+1}$ connect only to node $V_{-1}$, positive node $V_{q}^{+}$connect only to negative node $V_{q}^{-}$what right subscript is equal.

5) The power-function of node $V_{+1}$ is a constant +1 , the power-function of $V_{-1}$ is a constant -1 , the power-function of edge $E_{+1,-1}$ is a constant +2 , the flow-function of edge $E_{+1,-1}$ is a constant I;

6) In the graph $G_{K}$, other nodes and edges all be defined power-function $v_{i}(t)$, power-function $e_{i j}(t)$, flow-function $f_{i j}(t)$ and contradiction-function $R_{i j}(t)$, these are satisfied by Kirchhoff laws as above.

\subsection{Heredity and Variation Theorem}

1) For $t \in\left[t_{0}, t_{1}\right]$, if the 1-Order derivative of function $Q_{i j}(t)$ exist and if

$$
\frac{\mathrm{d} Q_{i j}(t)}{\mathrm{d} t} \neq 0
$$

then $Q_{i j}(t)$ is called variation, or not is called heredity.

2) Every contradiction-function $R_{i j}(t)$ always can be seen an algebra-sum of a constant $C$ and function $R_{i j}(t)$ i.e.

$$
R_{i j}(t)=Q_{i j}(t)+C(4)
$$

and if

$$
\frac{\mathrm{d} Q_{i j}(t)}{\mathrm{d} t} \neq 0
$$

then in Formulation (4) $Q_{i j}(t)$ is variation and $C$ is heredity.

3) Heredity and variation theorem

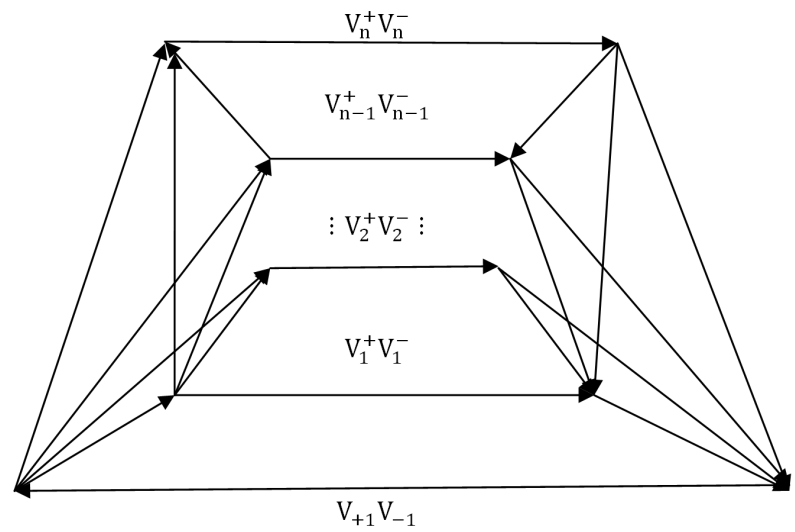

Figure 1. The structure of graph $G_{K}$. 
Heredity and variation is basic law in object-logic of cause in subject-logic.

Prove: combining the 11.1. and 11.2. as above which can be proved obviously.

\subsection{The Critical-Point Theorem}

For the contradiction-function $R_{i j}(t)$, if its m-order derivative exist and the points as below make

$$
\frac{\mathrm{d}^{k} R_{i j}(t)}{\mathrm{d} t^{k}}=0, \text { or } \infty, k=0,1, \cdots, m
$$

and some special-selected points are called critical-point.

\section{Property-Function Critical-Point Theorem}

The existence of critical-points will make what some new property-function is born or some old property-function is dead.

\subsection{Isomorphic-Equality $\leftrightarrow$}

If the rank-number of logic-variable $A_{i}^{ \pm s}(t), A_{j}^{ \pm r}(t)$ is equal i.e. $s=r$, then $A_{i}^{ \pm s}(t)$ and $A_{j}^{ \pm r}(t)$ is called isomorphic-equality, denote isomorphic-equality as

$$
A_{i}^{ \pm s}(t) \leftrightarrow A_{j}^{ \pm r}(t)
$$

Isomorphic-equality $\leftrightarrow$ is satisfied by as below reflexive law $A_{j}^{ \pm r}(t) \leftrightarrow A_{j}^{ \pm r}(t), A_{i}^{ \pm s}(t) \leftrightarrow A_{i}^{ \pm s}(t)$ symmetrical law if $A_{j}^{ \pm r}(t) \leftrightarrow A_{i}^{ \pm s}$, then $A_{i}^{ \pm s}(t) \leftrightarrow A_{j}^{ \pm r}(t)$ transtive law if $A_{i}^{ \pm s}(t) \leftrightarrow A_{j}^{ \pm r}(t)$ and $A_{j}^{ \pm r}(t) \leftrightarrow A_{m}^{ \pm k}(t)$, then $A_{i}^{ \pm s}(t) \leftrightarrow A_{m}^{ \pm k}(t)$

\subsubsection{Similarity between Logic-Variable $A_{i}^{ \pm s}(t)$ and $A_{j}^{ \pm r}(t)$,}

\section{Thinking Analogy Method}

1) Definition: the two logic-variable $A_{i}^{ \pm s}(t)$ and $A_{j}^{ \pm r}(t)$ is called similarity, if

a. $A_{i}^{ \pm S}(t) \leftrightarrow A_{j}^{ \pm r}(t)$

b. in the graph $G_{K}^{i}$ and $G_{K}^{j}$, their contradiction-function of corresponding edge is proportion i.e.

$$
R_{i j}^{i}(t)=C R_{i j}^{j}(t), R_{i j}^{i}(t) \in G_{K}^{i}, R_{i j}^{j}(t) \in G_{K}^{j}
$$

in formulation as above $\mathrm{C}$ is a constant.

2) If $A_{i}^{ \pm s}(t)$ and $A_{j}^{ \pm r}(t)$ is called similarity, what is denoted as $A_{i}^{ \pm s}(t) \sim A_{j}^{ \pm r}(t)$. The relation is satisfied as below reflexive law $A_{i}^{ \pm s}(t) \sim A_{i}^{ \pm s}(t), A_{j}^{ \pm r}(t) \sim A_{j}^{ \pm r}(t)$ symmetrical law if $A_{i}^{ \pm s}(t) \sim A_{j}^{ \pm r}(t)$, then $A_{j}^{ \pm r}(t) \sim A_{i}^{ \pm s}(t)$ transtive law if $A_{i}^{ \pm s}(t) \sim A_{j}^{ \pm r}(t)$, and $A_{j}^{ \pm r}(t) \sim A_{m}^{ \pm k}(t)$, then $A_{i}^{ \pm s}(t) \sim A_{m}^{ \pm k}(t)$

3) Thinking analogy theorem

Two of logic-variable $A_{i}^{ \pm s}(t)$ and $A_{j}^{ \pm r}(t)$ are analogy-able in thinking, if and only if 


$$
A_{i}^{ \pm s}(t) \sim A_{j}^{ \pm r}(t)
$$

Proof: based on the definition of similarity, if

$$
A_{i}^{ \pm s}(t) \sim A_{j}^{ \pm r}(t)
$$

then

$$
A_{i}^{ \pm s}(t)=C A_{j}^{ \pm r}(t)
$$

In formulation as above, $C$ is a constant, thus they are analogy-able in thinking.

Proof is Over.

\section{Conclusion}

As shown above, author has established an axiom system depending on several laws, some definitions, graph $\mathrm{G}_{\mathrm{K}}$ and Mozi-principle, and proved some theorems for dialectical logic K-model. The advanced properties and theorems for dialectical logic K-model will be explained in succedent papers by the author.

\section{References}

[1] Bencivenga, E. (2000) Hegel's Dialectical Logic. Oxford University Press, Oxford.

[2] Kosok, M. (1966) The Formalization of Hegel's Dialectical Logic. International Philosophical Quarterly, 6, 596-631. https://doi.org/10.5840/ipq19666456

[3] Johnston, I. (2010) The Mozi: A Complete Translation. The Chinese University Press, Hongkong.

[4] Berge, C. (1973) Graph and Hypergraphs. North-Holland Publishing Company, Amsterdam.

Submit or recommend next manuscript to OALib Journal and we will provide best service for you:

- Publication frequency: Monthly

- 9 subject areas of science, technology and medicine

- Fair and rigorous peer-review system

- Fast publication process

- Article promotion in various social networking sites (LinkedIn, Facebook, Twitter, etc.)

- Maximum dissemination of your research work

Submit Your Paper Online: Click Here to Submit

Or Contact service@oalib.com 